\title{
Solubility Enhancement of Clozapine through Co-crystal Formation with Isonicotinamide
}

\author{
Fikri Alatas $^{1 *}$, Hestiary Ratih ${ }^{1}$, Hesti Kurnia ${ }^{1}$, Sundani N. Soewandhi² \\ 1. Pharmaceutics group, Faculty of Pharmacy, Universitas Jenderal Achmad Yani, Jl. Terusan Jenderal \\ Sudirman, Cimahi, West Java, Indonesia, 40521 \\ 2. Pharmaceutics group, School of Pharmacy, Institut Teknologi Bandung, Jl. Ganesha 10, Bandung, \\ West Java, Indonesia.
}

Received: 17 Oct 2019/ Revised: 17 Nov 2019/ Accepted: 26 Nov 2019/ Published: 6 Jan 2020

\begin{abstract}
Clozapine (CLO) is an effective atypical antipsychotic to control the symptoms of psychosis and schizophrenia. Clozapine has low solubility and high permeability, so it is classified as a class II in the biopharmaceutical classification system. The aim of this study was to improve the solubility and dissolution rate of clozapine by clozapine-isonicotinamide (CLO-INA) co-crystal formation. CLOINA co-crystal was prepared by solvent-drop grinding (SDG) method using water as a solvent. Characterization of SDG result was conducted by powder X-ray diffraction (PXRD) and Fourier transform infrared (FTIR). Solubility test was performed in water at room temperature. The dissolution test was performed in $900 \mathrm{~mL}$ of $\mathrm{pH} 6.8$ phosphate buffer solution, 50 rotation per minute of paddle rotation, and at $37 \pm 0.5^{\circ} \mathrm{C}$. The PXRD pattern of SDG result of CLO-INA has many different peaks from its parent components, and this may indicate the co-crystal formation. The solubility of the cocrystal clozapine was fifteen folds higher than pure clozapine. The dissolution rate of CLO-INA cocrystal increased in the first 10 minutes compared to pure clozapine. Percentage of clozapine dissolved after 10 minutes from CLO-INA co-crystal and pure CLO were 10.2 and $2.4 \%$, respectively. CLO and INA can form co-crystal by SDG method that can improve the solubility and dissolution rate of clozapine.
\end{abstract}

Keywords: Clozapine, Isonicotinamide, Co-crystal, Solubility, Dissolution

\section{Introduction}

Clozapine (CLO) is a potent antipsychotic widely used to suppress both positive and negative symptoms of schizophrenia and some neuroleptic responses [1]. CLO is classified as a class II drug according to biopharmaceutics classification systems (BCS), due to its high permeability and low solubility [2]. The bioavailability of CLO is 27 to $50 \%[3]$.

Solubility is one of the critical physicochemical properties in predicting the degree of active pharmaceutical ingredients (APIs) absorption in the gastrointestinal tract. The very low solubility of API in water often indicates low bioavailability, consequently, the dissolution rate becomes the ratelimiting step in the drug absorption process [4].

The co-crystal formation is one of the alternative methods that can be used to increase solubility and dissolution rate in many APIs [5]. Co-crystal can

${ }^{\star}$ Corresponding author,

e-mail : fikri.alatas@lecture.unjani.ac.id (F. Alatas) be explained as complex of two or more neutral molecules bonded together in a crystal lattice via a non-covalent interaction, in particular, a hydrogen bond [6]. The formation of co-crystals involves combining drugs with pharmaceutically acceptable additives in the crystal lattice. The resulting multicomponent crystal form will have different physicochemical properties and can potentially improve solubility $[7,8]$, dissolution rate $[9,10]$, compressibility [11], chemical and physical stability [12].

The co-crystal formation depends on the functional group between the API and the co-crystal former (CCF) to allow the occurrence of hydrogen bonding. The presence of the -NH group on the diazepine ring in the CLO chemical structure (Fig. 1a) allows the co-crystals formation with carboxylic acid and amide groups. Isonicotinamide (INA) with the chemical structure shown in Fig. $1 \mathrm{~b}$ is one of 
the useful amide group compounds used in the formation of co-crystals and is known as the GRAS (Generally Recognized as Safe) compound. INA has a donor and some acceptor hydrogen bond that makes this CCF easy to form co-crystals with some APIs [13-17]. This purpose of this study was to know the influence of CLO-INA co-crystal formation on the solubility and dissolution rate of clozapine.

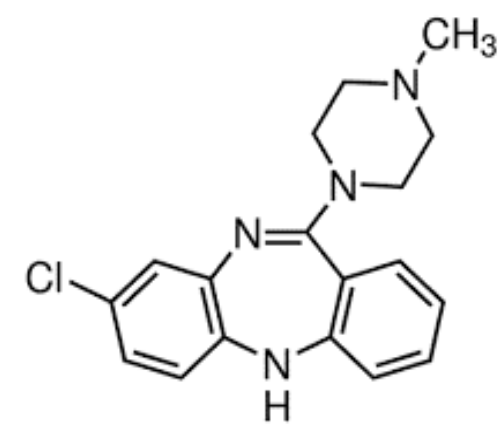

a<smiles>NC(=O)c1ccncc1</smiles>

b
Figure 1. Chemical structures of (a) clozapine dan (b) isonicotinamide

\section{Method}

\subsection{Materials}

The materials used in the present study were clozapine (Taizhou Xingming Pharmaceutical, China), isonicotinamide (Sigma-Aldrich), methanol (Merck), water, potassium dihydrogen phosphate (Merck), sodium hydroxide (Merck). While the tools used were powder X-ray diffractometer (Philips PW1710), Shimadzu FTIR Affinity-1 spectrophotometer(DRS-8000), orbital shaker(IKA KS-260), ultraviolet spectrophotometer (Shimadzu 1800PC), and TDTF ZRS-6G Dissolution tester.

\subsection{Solubility phase curve construction of CLO in} various concentration of INA solutions

A total of $50 \mathrm{mg}$ CLO was inserted into five vials containing five $\mathrm{mL}$ of an INA solution with a concentration range of 0.4 to $1.4 \mathrm{M}$ in an aqueous solvent and shaken with an orbital shaker at room temperature. After 24 hours, the samples were filtered. The filtrate was diluted and analyzed by Shimadzu 1800PC ultraviolet spectrophotometer at
$293 \mathrm{~nm}$. The test was carried out in triplicate. The phase solubility curve was constructed by plotting the level of dissolved CLO to the concentration of INA solution.

\subsection{Preparation of CLO-INA co-crystal by solvent-} drop grinding ( $S D G)$ method

An equimolar mixture of CLO (326 mg) and INA (122 mg) was milled simultaneously for five minutes in a mortar with the addition of two drops of water. The grinding result allowed to dry at room temperature.

\subsection{Characterization of CLO-INA SDG result by powder X-ray Diffraction method}

The SDG or wet grinding result of CLO-INA was characterized by Philips PW1710 powder $\mathrm{X}$-ray diffraction system and compared with pure CLO and INA powder X-ray diffraction patterns. The powder X-ray diffraction data collection was performed by scanning 100-200 mg of sample powder placed on a glass container and flattened at an angle range of $2 \theta=5-45^{\circ}$, scan rate $2 \% \mathrm{~min}$, with a voltage of $40 \mathrm{kV}$, and a current of $30 \mathrm{~mA}$.

\subsection{Characterization of CLO-INA SDG result by} Fourier transform infra-red (FTIR)

The FTIR spectral data collection of CLOINA wet grinding result was performed using the Shimadzu FTIR Affinity-1 spectrophotometer (DRS-8000) and compared with CLO and INA pure spectra. The samples were homogeneously mixed with potassium bromide at a weight ratio of 1:5 and scanned in the 4000-400 cm-1 wavenumbers range. $\mathrm{KBr}$ powder was used as a blank for background correction.

\subsection{Solubility test}

As much as $50 \mathrm{mg}$ of CLO-INA wet grinding result and pure CLO were placed into vials that containing each of five $\mathrm{mL}$ of water and shaken on an orbital shaker (IKA KS-260) at room temperature. After 24 hours, the samples were filtered, and the filtrate was analyzed by ultraviolet 
spectrophotometer at a wavelength of $293 \mathrm{~nm}$.

\subsection{Dissolution test}

The dissolution test was carried out on SDG product of CLO-INA and pure CLO in a $900 \mathrm{~mL}$ pH 6.8 of phosphate buffer solution at $37 \pm 0.5^{\circ} \mathrm{C}$ using a type I apparatus with a rotation speed of 50 rotations per minute (rpm) for 60 minutes. The dissolved clozapine in each sample was diluted and analyzed by ultraviolet spectrophotometer at a 293 $\mathrm{nm}$. The test was performed in six replicate.

\section{Result and Discussion}

\subsection{Solubility phase curve of CLO-INA}

The drug solubility study in a CCF solution can be used to predict the formation of co-crystals by making a phase solubility curve. The phase solubility curve is the solubility curve that describes the concentration of a solute in the concentration variation of another substance solution. According to Higuchi and Connors, there are several types of phase solubility curves, namely $\mathrm{AL}, \mathrm{AP}, \mathrm{AN}$, BS, dan BI types [18]. The phase solubility test is useful to know the type of solubility curve of CLO in the variation of INA concentration, and this can be an indication of the occurrence of the cocrystal formation. Determination of dissolved CLO was carried out by ultraviolet spectrophotometry method. The CLO has three maximum absorption wavelengths, namely 230, 260, and $293 \mathrm{~nm}$. INA still has absorption at wavelengths of 230 and 260 $\mathrm{nm}$, but has no absorption at $293 \mathrm{~nm}$ wavelength, so a wavelength of $293 \mathrm{~nm}$ is used to analyze CLO concentration without interference by INA. Fig. 2 showed the CLO phase solubility curve in the concentration variation of the INA solution in water follows the BS type. The CLO solubility increase sharply at INA concentrations of 0.4 to $1 \mathrm{M}$ caused by the co-crystal began to form with high solubility in water. At INA concentration of 1-1.4 M, CLO solubility decreased due to the achievement of CLO-INA co-crystal solubility product constant (Ksp) in water, accordingly, CLO-INA co-crystal solid was formed. The higher concentration of
INA, the more co-crystal solid was formed. This is in accordance with the explanation of Mantri et al., 2009 [19].

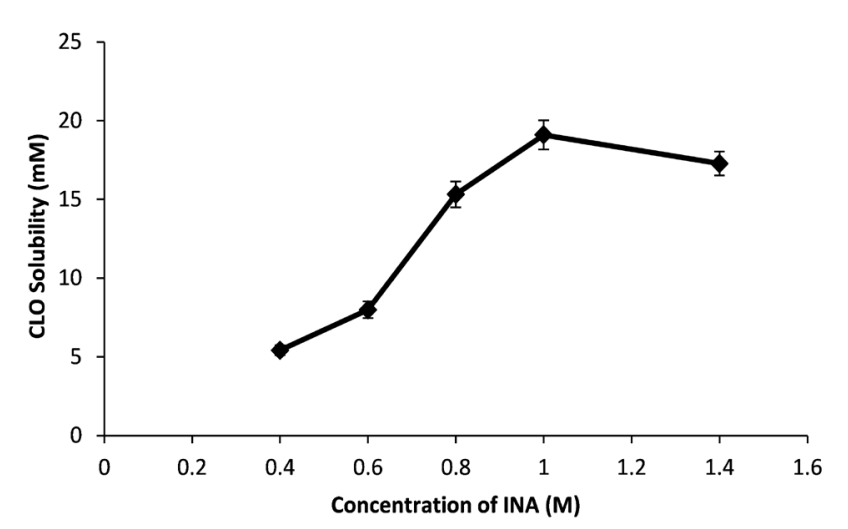

Figure 2. Phase solubility curve of clozapine (CLO) in the various concentration of isonicotinamide (INA) solution in water. $(\mathrm{n}=3)$

3.2 Preparation of CLO-INA co-crystal by solventdrop grinding (SDG) method

Physical interaction can occur when two substances are milled simultaneously. The interaction may be a mixture of eutectic or the formation of new molecular compound or cocrystal. In this experiment, an equimolar mixture of CLO and INA was milled with the addition of two drops of water, and this method known as wet grinding or solvent-drop grinding (SDG) [20]. The purpose of solvent addition is to accelerate the achievement of the amorphous state of the two components causing each component to be more reactive and faster co-crystallization [21]. At least, the solvent can dissolve well one of the components. Water is used as a solvent because it can dissolve well INA and slightly CLO.

\subsection{Characterization of CLO-INA SDG result by} powder X-ray Diffraction method

The initial characterization of interaction in the wet milling process is by using powder X-ray diffraction (PXRD). The different of PXRD pattern of SDG result from PXRD patterns of their respective constituent components may indicate the co-crystal formation. The PXRD pattern of CLO- 
INA compared to its parent components (pure CLO and INA) is shown in Fig. 3. The PXRD pattern of CLO shows the main peaks at the angle of $2 \theta=$ $10.6 ; 17.5 ; 19.4 ; 21.2$; and $29.9^{\circ}$. These results are consistent with those reported by the previous study [22]. The INA raw material has the main peaks at an angle of $2 \theta=17.9 ; 20.9 ; 23.4 ; 26.0$; and $31.1^{\circ}$, and this corresponds to INA form I with reference code EHOWIH01 [23]. The powder X-ray diffraction pattern of CLO-INA wet grinding result shows the formation of new peaks that are different from the main peaks of CLO and INA. The differences of PXRD pattern between CLO-INA SDG result and its pure components indicate the formation of CLO-INA co-crystal. The main peaks of CLO-INA co-crystal are located at an angle of $2 \theta=11.1 ; 13.6$; $17.9 ; 24.3$; and $29.3^{\circ}$.

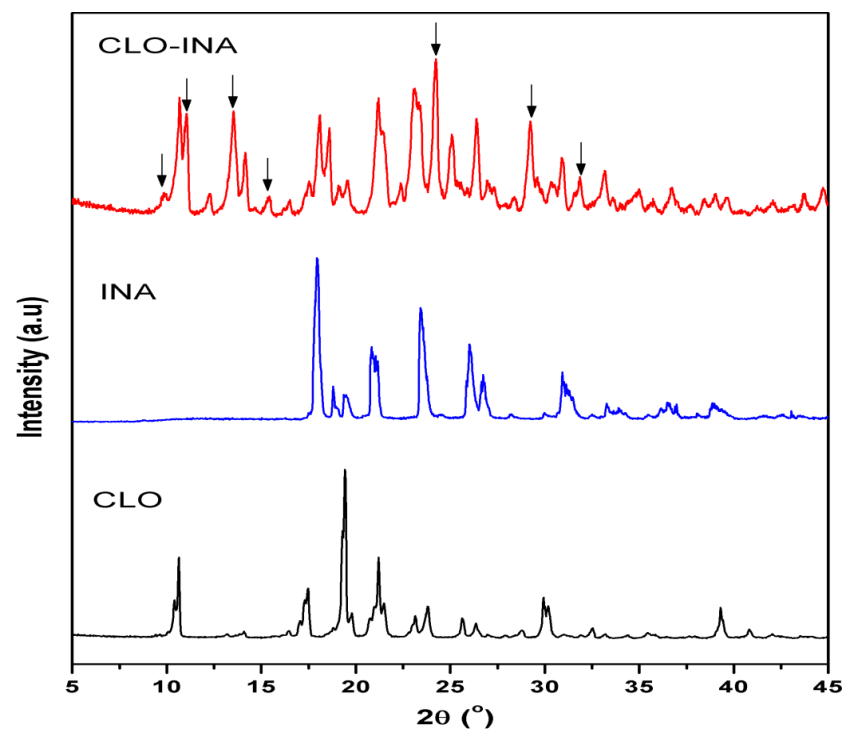

Figure 3. Powder X-ray diffraction patterns of CLO, INA, and CLO-INA

\subsection{Characterization of CLO-INA SDG result by} Fourier transform infra-red (FTIR)

One spectroscopic technique that can be used to show the formation of co-crystal is FTIR. The presence of shifts in the number of spectral waves in certain groups, such as carboxylates $(-\mathrm{COOH})$, carbonyl $(\mathrm{C}=\mathrm{O})$, amines $\left(-\mathrm{NH}_{2}\right)$, imines $(-\mathrm{C}=\mathrm{NH})$ can indicate the presence of hydrogen bonds between two interacting components [24]. Fig. 4 showed the FTIR spectrum of the CLO-INA
SDG result compared with their pure constituent components. The CLO has two sharp peaks at 3294 and $1594 \mathrm{~cm}^{-1}$ caused by stretching vibration of -NH and amidine $(\mathrm{C}=\mathrm{N})$ group, respectively. The INA has two peaks at 3368 and $3186 \mathrm{~cm}^{-1}\left(-\mathrm{NH}_{2}\right.$ group stretching vibration) and a peak at $1668 \mathrm{~cm}-1$ (carbonyl group vibration). The FTIR spectrum of the CLO-INA wet grinding result indicated the peaks of -NH group and amidine group of CLO shifted to 3298 and $1597 \mathrm{~cm}^{-1}$, respectively, as well as the carbonyl group vibration of INA shifted to $1678 \mathrm{~cm}^{-1}$. This shifting was thought caused by the hydrogen bond between the -NH group of CLO and the $\mathrm{C}=\mathrm{O}$ group of INA that indicated the CLO-INA co-crystal formation.

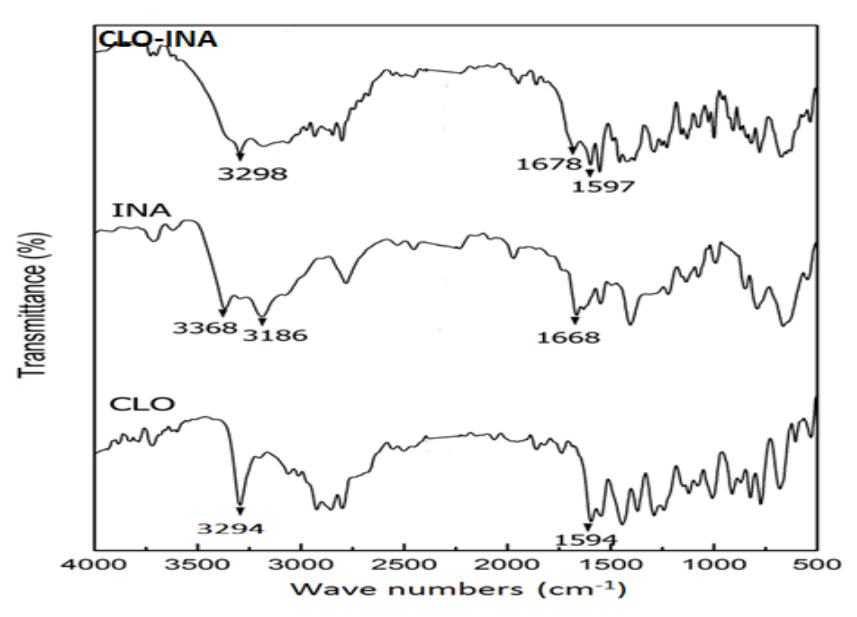

Figure 4. FTIR spectrum of CLO-INA co-crystal compared to its pure components.

\subsection{Solubility of CLO-INA Co-crystal}

Solubility is an important physicochemical factor which may indicate a difference in the crystalline structure of a compound, such as a polymorph, salt, hydrate, solvate, and co-crystalline [25]. The solubility test was performed to determine the change of clozapine solubility in consequence of CLO-INA co-crystal formation. The result of the water solubility test at room temperature showed CLO-INA co-crystal has a solubility of $378 \pm 12$ $\mu \mathrm{g} / \mathrm{mL}$, which is 15 -folds higher than pure CLO solubility $(24 \pm 1 \mu \mathrm{g} / \mathrm{mL})$. This solubility increasing was due to the creation of hydrogen bonds between CLO and INA that changes in the crystal structure. 
In the dissolution process of a solid in a solvent, intermolecular bond termination between the solute and the solvent requires energy [26]. The energy required to break the bonds between dissolved solid depends on the structure of the solid compound and the intermolecular attraction between the dissolved solid compounds, therefore the existence of hydrogen bonds in CLO-INA co-crystal can reduce the energy required to break the bonds between CLO-INA co-crystal and water so that the CLOINA co-crystal has a higher solubility than pure CLO.

\subsection{Dissolution profile of CLO-INA Co-crystal}

The dissolution rate is also a physicochemical property which can show the difference in the crystalline solids structure. An increasing in dissolution rate follows the increasing of solubility. The BCS class II drug will be absorbed well in the gastrointestinal tract when the dissolution rate of the drug is high. CLO has a low solubility at neutral $\mathrm{pH}$, and its solubility increases with decreasing $\mathrm{pH}$ due to the ionization. In this study dissolution test carried out at $\mathrm{pH} 6.8$ which represents the $\mathrm{pH}$ conditions of the intestinal tract. The result of the particulate dissolution test in phosphate buffer media pH 6.8 (Fig. 5) shows the dissolved percentage of clozapine after $10 \mathrm{~min}$ (DP10) from the CLO-INA and CLO pure are 10.24 and $2.4 \%$, resnestively and this shows that the CI O-INA co-

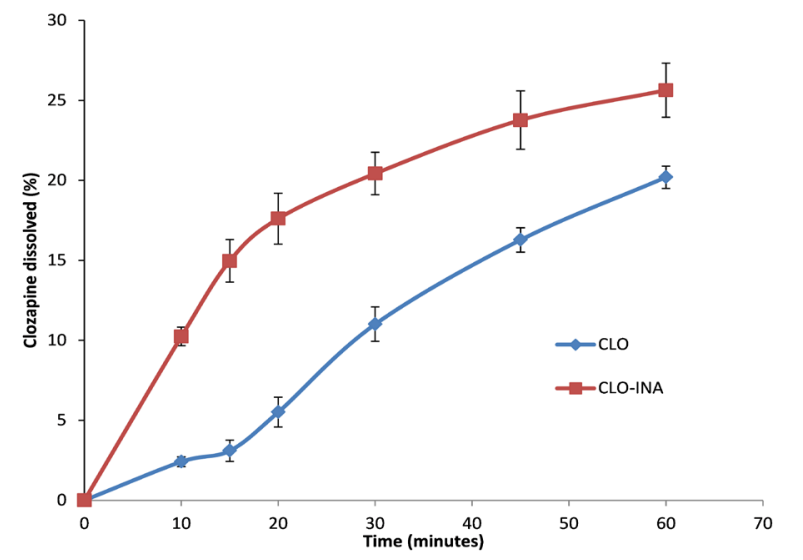

Figure 5. Profile dissolution of CLO-INA co-crystal in
pH 6.8 of phosphate buffer solution compared to pure CLO. $(\mathrm{n}=6)$

\section{Conclusion}

Clozapine and isonicotinamide may form cocrystal by a solvent-drop grinding method with the addition of water solvent based on characterization results by powder X-ray diffraction, Fourier transforms infrared, and solubility phase curve methods. The formation of CLO-INA co-crystal can enhance the solubility and dissolution rate of clozapine.

\section{Acknowledgements}

We would like to thank the Research and Community Services (LPPM) of Universitas Jenderal Achmad Yani for the support of research grant.

\section{References}

[1] Manu P, Sarpal D, Muir O, et al. When can patients with potentially life-threatening adverse effects be rechallenged with clozapine? A systematic review of the published literature. Schizophr Res 2012; 134: $180-186$.

[2] Dias SBT, Nascimento TG, Santos AFO, et al. Polymorphic characterization and compatibility study of clozapine: implications on its stability and some biopharmaceutics properties. J Therm Anal Calorim 2014; V.12x: X1.

[3] Stahl SM. Antipsychotics and mood stabilizer. 3rd ed. New York: Cambridge University Press, 2008.

[4] Chadha R, Saini A, Jain DS, et al. Preparation and solid-state characterization of three novel multicomponent solid forms of oxcarbazepine: Improvement in solubility through saccharin cocrystal. Cryst Growth Des 2012; 12: 42114224.

[5] Babu NJ, Nangia A. Solubility advantage of amorphous drugs and pharmaceutical cocrystals. Cryst Growth Des 2011; 11: 26622679.

[6] Rodríguez-Hornedo N, Nehm SJ, Jayasankar A. Cocrystals: design, properties and formation mechanisms. Encycl Pharm 
Technol 2007; 615-635.

[7] Alatas F, Ratih H, Soewandhi SN. Enhancement of solubility and dissolution rate of telmisartan by telmisartan-oxalic acid co-crystal formation. Int J Pharm Pharm Sci 2015; 7: 423-425.

[8] McNamara DP, Childs SL, Giordano J, et al. Use of a glutaric acid cocrystal to improve oral bioavailability of a low solubility API. Pharm Res 2006; 23: 1888-1897.

[9] Tomaszewska I, Karki S, Shur J, et al. Pharmaceutical characterisation and evaluation of cocrystals: Importance of in vitro dissolution conditions and type of coformer. Int J Pharm 2013; 453: 380-388.

[10] Zegarac M, Leksic E, Sket P, et al. A sildenafil cocrystal based on acetylsalicylic acid exhibits an enhanced intrinsic dissolution rate. CrystEngComm 2014; 16: 32-35.

[11] Aher S, Dhumal R, Mahadik K, et al. Effect of cocrystallization techniques on compressional properties of caffeine/oxalic acid 2:1 cocrystal. Pharm Dev Technol 2011; 1-6.

[12] Babu NJ, Sanphui P, Nangia A. Crystal engineering of stable temozolomide cocrystals. Chem - An Asian J 2012; 7: 2274 2285.

[13] Wang L, Tan B, Zhang H, et al. Pharmaceutical cocrystals of diflunisal with nicotinamide or isonicotinamide. Org Process Res Dev 2013; 17: 1413-1418.

[14] Dubey R, Desiraju GR. Structural landscape of the $1: 1$ benzoic acid:isonicotinamide cocrystal. Chem Commun 2014; 50: 11811184.

[15] Madeley LG, Levendis DC, Lemmerer A. Isonicotinamide-2-naphthoic acid (1/1). Acta Crystallogr Sect E Struct Reports Online; 67.

[16] Báthori NB, Lemmerer A, Venter GA, et al. Pharmaceutical co-crystals with isonicotinamide_vitamin B3, clofibric acid, and diclofenac - and two isonicotinamide hydrates. Cryst Growth Des 2011; 11: 75-87.

[17] Sanphui P, Kumar SS, Nangia A.
Pharmaceutical cocrystals of niclosamide. Cryst Growth Des 2012; 12: 4588-4599.

[18] Takeru Higuchi, Kenneth A. Connors. Phase solubility techniques. In: Advances in Analytical Chemistry and Instrumentation. New York: Jonh Wiley \& Sons, INC, 1965, pp. 117-212.

[19] Mantri RV, Sanghvi R, Zhu H (Jim). Solubility of Pharmaceutical Solids. In: Yihong Qiu, Yisheng Chen, Geoff G.Z. Zhang, Lirong Liu WRP (ed) Developing Solid Oral Dosage Forms: Pharmaceutical Theory And Practice. New York: Academic Press, 2009, pp. 1-24.

[20] Trask A V, Motherwell WDS, Jones W. Solvent-drop grinding: green polymorph control of cocrystallisation. Chem Commun (Camb) 2004; 890-891.

[21] Rehder S, Klukkert M, Löbmann K a M, et al. Investigation of the formation process of two piracetam cocrystals during grinding. Pharmaceutics 2011; 3: 706-722.

[22] Mahmoud A, Ali A, Ali AA, et al. Clozapinecarboxylic acid plasticized co-amorphous dispersions: Preparation, characterization and solution stability evaluation. 2015; 65 : 133-146.

[23] Aakeröy CB, Beatty AM, Helfrich BA, et al. Do polymorphic compounds make good cocrystallizing agents? A structural case study that demonstrates the importance of synthon flexibility. Cryst Growth Des 2003; 3: 159165.

[24] Qiao N, Li M, Schlindwein W, et al. Pharmaceutical cocrystals: An overview. Int J Pharm 2011; 419: 1-11.

[25] Sarma B, Chen J, Hsi HY, et al. Solid forms of pharmaceuticals: Polymorphs, salts and cocrystals. Korean J Chem Eng 2011; 28: 315-322.

[26] Saikia B, Bora P, Khatioda R, et al. Hydrogen Bond Synthons in the Interplay of Solubility and Membrane Permeability/Diffusion in Variable Stoichiometry Drug Cocrystals. Cryst Growth Des 2015; 15: 5593-5603. 